\title{
Enfermagem frente à reabilitação de pacientes submetidos à amputação
}

\author{
Daiane Mariano ${ }^{1}$ \\ Sandra Maria Cezar Leal ${ }^{2}$ \\ Joannie dos Santos Fachinelli Soares ${ }^{3}$ \\ Greice Kelli Celho de Souza ${ }^{4}$
}

Resumo: investigar como a enfermagem enfrenta o cuidado ao paciente submetido à amputação de extremidades e quais as ações que contribuem para reabilitação após a alta hospitalar. Método: estudo qualitativo, análise temática. Questões norteadoras: Como a enfermagem enfrenta o cuidado ao paciente com amputação? Os cuidados durante a hospitalização podem contribuir para reabilitação após a alta hospitalar? Coleta com entrevistas gravadas, com 10 profissionais da equipe de enfermagem de uma rede hospitalar pública, Porto Alegre/RS. Porto Alegre/RS. Resultados: na análise emergiram as duas categorias: características dos pacientes amputados; A equipe de enfermagem frente ao cuidado do paciente amputado. Conclusão: a criação de protocolo sistematizado de atendimento e a revisão do sistema de suporte no pós-alta, pode contribuir para a reabilitação da pessoa amputada. Descritores: Amputação; Enfermagem; Hospitalização; Cuidados de Enfermagem.

\section{Nursing facing rehabilitation of patients submitted to amputation}

Abstract: investigate how nursing care faces the care to the patient with amputation of the extremities and which actions contribute for the re-habilitation after the hospital discharge. Method: qualitative study and thematic analysis. Guiding questions: How does nursing face the care to the patient with amputation? Can the care given during the hospitalization contribute for the re-habilitation after the hospital discharge? Collection with recorded interviews, signature of free and cleared up consent statements, in October 2011 , with 10 professionals of the nursing staff from a public hospital network, Porto Alegre-RS. Results: two categories derived from the analysis: Characteristics of the amputated patients; The nursing staff facing the care to the amputated patient. Conclusion: the creation of a systematized attendance protocol and the revision of the support system in the post-discharge can contribute for the amputated subject re-habilitation.

Descriptors: Amputation; Nursing; Hospitalization; Nursing Care.

\section{Enfermería ante la rehabilitación de pacientes sometidos a amputación}

Resumen: investigar como la enfermería enfrenta el cuidado al paciente sometido a amputación de extremidades y las acciones que contribuyen para la rehabilitación tras el alta hospitalaria. Método: estudio cualitativo, análisis temático. Cuestiones orientadoras: ¿Cómo la enfermería enfrenta el cuidado al paciente con amputación? ¿Pueden los cuidados durante la hospitalización contribuir para la rehabilitación tras el alta hospitalaria? Recolección con entrevistas grabadas, firma de declaraciones de consentimientos libres y esclarecidos, en octubre 2011 , con 10 profesionales del equipo de enfermería de una red hospitalaria pública, Porto Alegre-RS. Resultados: del análisis surgieron dos categorías: Características de los pacientes amputados; El equipo de enfermería frente al cuidado del paciente amputado. Conclusión: creación de protocolo sistematizado de atendimiento y la revisión del sistema de Descriptores: Amputación; Enfermería; Hospitalización; Cuidados de Enfermería.

\section{INTRODUÇÃO}

A mputação é a retirada de parte do corpo, geralmente de um membro'. Além de traumas, algumas doenças figuram como responsáveis pela necessidade de remoção de membros, como Diabetes Mellitus e doenças ateroscleróticas. Diante deste agravo, a discussão acerca do processo de reabilitação de pacientes amputados revela-se atual, devendo ocupar espaço de destaque entre os profissionais de saúde².

O cuidado às pessoas amputadas exige competências específicas, desenvolvidas com base nas políticas públicas e com a disponibilidade de estrutura física e de serviços de referência e contra-referência. Nesse sentido, a equipe de enfermagem desempenha papel importante nos cuidados a esses pacientes, tanto no preparo pré-operatório como nos cuidados imediatos pós-amputação, com orientações ao paciente e familiares visando à reabilitação ${ }^{3}$.

Assim, a justificativa do tema escolhido fundamenta-se na relevância do problema. Pois, a vítima de amputação experimenta barreiras para retorno ao convívio social, que podem ser mais facilmente superadas com a rápida identificação de sintomas que sinalizem retrocessos biofísicos e psicossociais.
Nessa perspectiva, o objetivo desta pesquisa foi investigar como a enfermagem enfrenta o cuidado ao paciente com amputação de extremidades e quais as ações que contribuem para reabilitação após a alta hospitalar.

\section{METODOLOGIA}

Estudo qualitativo e exploratório, realizado na Unidade Vascular de uma rede hospitalar pública, em Porto Alegre/RS, cujo serviço é especializado no tratamento de pacientes com problemas vasculares. Participaram profissionais da equipe de enfermagem envolvidos no cuidado ao paciente amputado, durante a hospitalização. Foram incluídos os enfermeiros, auxiliares e técnicos de enfermagem, que exerciam suas atividades na unidade hospitalar em estudo. Excluídos os que estavam afastados por férias ou licença de saúde, no período da coleta dos dados. $\mathrm{O}$ aceite em participar da pesquisa e a saturação dos dados foram os critérios para determinar o número de participantes ${ }^{4}$. Dessa forma, o estudo contou com 10 profissionais: cinco auxiliares de enfermagem, um técnico de enfermagem e quatro enfermeiras. 
A coleta foi realizada no local de trabalho, por meio de entrevista semiestruturada, com as seguintes questões norteadoras: como a enfermagem enfrenta o cuidado ao paciente com amputação? Os cuidados durante a hospitalização podem contribuir para reabilitação após a alta hospitalar. Para garantir o anonimato, eles foram identificados como: Aux1, Aux2, Tec1, Tec2, E1, E2... Os profissionais de enfermagem foram esclarecidos sobre os objetivos do estudo, preservação do anonimato e assinaram o Termo de Consentimento Livre e Esclarecido. A análise dos dados foi realizada por meio da técnica de análise temática ${ }^{4}$. Como ferramenta de apoio utilizou-se o software NVivo.

Seguiram-se as normas preconizadas na Resolução 196/1996 do Conselho Nacional de Saúde5. O projeto de pesquisa foi aprovado pelo Comitê de Ética e Pesquisa da instituição em estudo, por meio do parecer $n^{\circ} 11-059$.

\section{RESULTADOS}

Entre os participantes deste estudo, $80 \%$ são do sexo feminino. A faixa etária variou entre 29 e 60 anos, sendo que $80 \%$ dos participantes situavam-se na faixa de 40 e 50 anos; $60 \%$ exercem a profissão há mais de 20 anos, sendo que o tempo de atividade com pacientes amputados variou de menos de um a 17 anos; $80 \%$ dos participantes trabalham há menos de 10 anos em unidades de amputados.

Após a análise das entrevistas construíram-se duas categorias: "Características dos pacientes amputados" e"Equipe de enfermagem frente ao cuidado do paciente amputado".

Na categoria Características dos Pacientes Amputados, identifica-se traços do perfil psicológico dos pacientes internados. É mencionada a dificuldade de aceitação pelo paciente da realidade de amputação. Essa dificuldade resulta em comprometimento emocional e na resistência ao tratamento, o que é expresso pelos profissionais:

É um paciente que requer maior atenção. [...] tem certa resistência, fica mais sensível e requer mais cuidado". (Aux 1.fem)

Tem paciente que se revolta, que não aceita. Alguns se tornam agressivos, então dificulta o atendimento, porque tu não consegues atendê-lo adequadamente[...]. (E4.fem)

Considerando o comprometimento emocional do paciente é importante que o profissional destacado para atendê-lo, além dos cuidados com os aspectos físicos, esteja atento ao apoio psicológico. O que é ilustrado na fala da enfermeira:

Em relação ao cuidado do paciente amputado acho que a gente tem que começar pela parte primordial, que é o apoio psicológico [...]. (E1.fem)

Ainda, sinalizado como característica dos pacientes amputados, destaca-se o relato sistemático das dores físicas, que segundo os profissionais, são fortes, constantes e constituem fator que dificulta o tratamento: a dor é tão intensa, tão importante que ele pede para ser amputado. (E1.fem)

Diversos entrevistados depuseram acerca de reclamações por parte de pacientes relacionadas à existência de dores no membro amputado, após a amputação, a chamada dor fantasma. [...] a dor fantasma [...] continuam verbalizando dor na extremidade amputada. [...] a queixa é de dor no pé (amputado). (E1.fem)
Ainda na mesma categoria pode-se identificar a característica nomeada dificuldades motoras, como uma resposta frequente ao questionamento sobre as dificuldades enfrentadas pelos pacientes durante os cuidados de enfermagem.

A dificuldade maior é quando a pessoa ainda deambulava [...] aí tem aquela limitação motora [...]depender de outras pessoas. (E3.masc)

Outro aspecto, relacionado ao perfil dos pacientes, reporta-se à reinternação hospitalar para fazer novo procedimento ou amputação. O que dificulta é que o paciente acaba fazendo um tratamento e não termina. [...] retorna fazer amputação, com necrose e infecção da ferida operatória. (Aux 1.fem)

Segundo os entrevistados, frequentes internações nomeadas como idas e vindas podem influenciar negativamente na estruturação psicológica dos pacientes. Outra dificuldade na aceitação é que eles vão amputando aos poucos [...] o dedo, daí a pouco o ante pé, [...] por fim amputam toda a perna. (E2.fem)

Na categoria denominada Equipe de enfermagem frente ao cuidado do paciente amputado, descreve-se os cuidados com o paciente. Nos quais, destaca-se o viés dos profissionais acerca das suas próprias atuações e das demais condições de atendimento aos pacientes.

O cuidado do paciente é muito bom, [...] poderia ser melhor se tivesse todos os materiais, pois sempre falta alguma coisa, um exemplo, são as roupas de cama que chegam muitas vezes no final do plantão. (Aux 3.fem)

Como se depreende pelo depoimento, sob a ótica do profissional, o atendimento pessoal dispensado aos pacientes é satisfatório, sendo que as condições de trabalho e os processos de trabalho poderiam ser melhorados. Porém, um entrevistado manifestou o entendimento de que sua atuação pessoal poderia ser melhor (mais humana).

A parte prática é bem feita, bem executada, [...] às vezes, não se tem tempo de conversar e de explicar algumas coisas para ele. (Téc1.fem)

As orientações prestadas aos pacientes foi outro aspecto sinalizado pelos profissionais. Pelos depoimentos verificou-se que a orientação do pós-operatório é compartilhada pelo médico e pela enfermeira, dentro de suas especialidades, no momento da alta, visando preparar o paciente para a vida fora do hospital.

Referenciaram, também, a possibilidade de apoio por psicóloga e assistente social, demonstrando a disponibilidade de uma equipe multiprofissional. "Nos dois últimos meses tem uma psicóloga que vem acompanhando os pacientes. O que sempre foi solicitado pelo serviço". (E1.fem)

Os entrevistados foram unânimes em destacar a existência do Programa de Assistência Domiciliar (PAD), relacionada com a vida dos pacientes fora do hospital, como ferramenta multidisciplinar de apoio ao paciente. 
[...] é encaminhado para um grupo de referência que é o PAD. É a assistente social que encaminha. [...] o paciente tem que residir na área de abrangência do PAD. [...] o programa dá assistência quanto a curativos, medicações, acompanhamento no domicilio [...]. (Aux 1.fem)

Os profissionais reportaram-se às dificuldades enfrentadas, enfatizando a deficiência de infraestrutura que encontram no cotidiano.

O banheiro não tem as barras de proteção, as cadeiras não são adequadas para levar o paciente ao banho, a cama, às vezes, não tem proteção. [...], acho que tem que melhorar isso. (Aux 2.fem)

Outra dificuldade mencionada pelos profissionais de enfermagem é que apesar dos pacientes amputados serem pessoas com particularidades específicas, inexiste um protocolo de atendimento desenvolvido para o cuidado à pessoa amputada.

[...] cada um faz o que acha melhor [...] até para o cuidado no pré e pós não tem protocolo. [...] é mais um aprimoramento de técnicas, [...], então agente tem aquela coisa do dia a dia, mas não uma coisa que seja específica para esse paciente amputado o que seria extremamente necessário. (E1.fem)

\section{DISCUSSÃO}

Os profissionais de enfermagem envolvidos no atendimento de pacientes submetidos à amputação, no hospital eleito para pesquisa, caracterizam-se pela dedicação às suas atribuições. É evidente a preocupação com o bem-estar dos pacientes, apesar de, muitas vezes, a estrutura física prejudicar o atendimento almejado.

Os depoimentos dos entrevistados foram uníssonos em afirmar que os pacientes amputados são pessoas com alteração da sensibilidade emocional, dor física e dificuldades motoras. Acerca do aspecto emocional, não é exagero afirmar que a cirurgia para retirada de um membro pode afetar mais emocionalmente do que uma cirurgia interna. Isso se dá porque, a amputação de um membro é visível, lembrando o paciente, dia após dia, de sua sensação de perda. Apesar de existirem poucas pesquisas sobre os efeitos da amputação nos anos iniciais após a cirurgia (até dois anos), tais estudos evidenciam sintomas depressivos durante a hospitalização e imediatamente após a amputação ${ }^{6}$.

Perder uma parte do corpo pode significar ter a existência alterada, viver um vazio que acarreta uma séria de mudanças na vida da pessoa. Significa ter que adaptar-se e readaptar-se, aprender a viver de novo, aceitar outra perspectiva no mundo ${ }^{7}$. Porém, a amputação também pode gerar sentimentos ambíguos, "ora de tristeza pela perda de parte de seu corpo, ora de alívio da dor e desconfortos provocados pelo odor"8.

Verificou-se, pela análise dos depoimentos, que os profissionais de enfermagem (aqui compreendidos os enfermeiros, técnicos e auxiliares), experimentam, no dia a dia, algumas dificuldades no atendimento aos pacientes. A falta de infraestrutura de suporte ou de dimensionamento insuficiente de profissionais interfere, negativamente, no cenário de atendimento, mesmo com o empenho e dedicação dos profissionais envolvidos. Ainda assim, esforços têm sido empregados pelos gestores do sistema de saúde que buscam a melhoria do atendimento em saúde no pais ${ }^{9}$.

A alteração da sensibilidade emocional do paciente deve ser monitorada pelos profissionais responsáveis por atendê-lo. Além dos cuidados com os aspectos físicos, é importante detectar outras circunstâncias que poderão prejudicar o tratamento, como variações de humor, sinais de depressão, resistência da família, entre outros. Assim, no "processo de reestruturação para viver a nova realidade o enfermeiro terá o papel essencial para o despertar, além da dimensão biológica, do sujeito e sua família"8:537.

O paciente que percebe sua situação como uma nova condição, parece ter mais sucesso no processo de adaptação, pois consegue pensar e conviver com a limitação, não como sinônimo de inutilidade ou de dependência total, mas como a superação de risco de morte (nos acidentes) ou de alivio de dores (nas doenças crônicas) ${ }^{10}$. Perder parte do corpo é doloroso e impõe um novo modo de viver, de estar no mundo e se relacionar com ele, exigindo um redimensionar, pois o corpo foi alterado e, consequentemente, a percepção do mundo e das coisas ${ }^{11}$.

O Hospital onde se realizou a pesquisa está direcionando suas ações estratégicas para a criação de um ambiente favorável à recuperação dos pacientes amputados. Segundo os depoimentos, recentemente foi contratada uma psicóloga que, no âmbito de sua especialidade, trabalha para complementar o atendimento oferecido por médicos e profissionais de enfermagem. Esta inclusão contribui para o estabelecimento de condições satisfatórias para o cuidado humanizado do paciente ${ }^{12}$.

No aspecto de investimento em infraestrutura, a preocupação mais relatada foi acerca das condições de suporte para pacientes com dificuldades motora. Claramente, há necessidade de uma maior atenção aos detalhes estruturais que facilitem a movimentação de pacientes amputados. Esse aspecto é importante, pois pode reduzir o grau de dependência dos pacientes, contribuir para melhoria de sua autoestima. A existência de condições que contribuam para o atendimento de qualidade é essencial quando se trata de pacientes amputados e, portanto, dotados de particularidades específicas 13

A pre]ocupação com a qualidade na prestação de serviços de saúde não é recente e o objetivo do cliente ao necessitar dos serviços hospitalares é de restabelecer sua saúde, solucionar problemas e equilibrar as disfunções. Para que ele possa usufruir de serviço de qualidade é necessário sistema gerencial que reconheça as suas necessidades, estabeleça padrões e busque mantê-los para assegurar a sua satisfação ${ }^{14}$

Conforme se percebe, o cuidado com as condições físicas, também, é relevante na reabilitação do paciente. $E$ a especial atenção ao tratamento pós-amputação é importante, pois esta fase define a velocidade de reabilitação do paciente. Assim, os objetivos principais dessa etapa devem ser: prevenir as contraturas articulares; fortalecer e coordenar o controle muscular do membro amputado; fortalecer e mobilizar a perna não afetada; fortalecer e mobilizar o tronco; controlar o edema do coto; estimular a independência; e, no caso de membros inferiores, estimular a deambulação precoce com auxiliares de marcha².

Em relação ao acompanhamento no pós-alta dos pacientes deste estudo, verifica-se que, apesar de frequentes menções à existência do Programa de Assistência Domiciliar (PAD), a referência às reinternações causadas por complicações supervenientes foi muito alta. E esse é um ponto que deveria ser mais observado e estudado, buscando-se a identificação das causas, para adoção de medidas preventivas por pacientes e profissionais da saúde.

Possivelmente os profissionais do PAD, não conseguem interferir nas situações mais graves, mas contribuem para a identificação precoce dos pacientes que não estão seguindo adequadamente as orientações da alta hospitalar, na condição de amputado. Assim, complicação com o coto e a não adaptação à situação de amputado são outros fatores que parecem influenciar no alto número de retornos. 
O reforço das atividades de instrução aos pacientes e familiares acerca dos cuidados necessários para sua recuperação pode contribuir para a redução do número de re-internações. Destaca-se a importância de a enfermagem orientar o cuidador de pessoas em condição crônica, visando qualificar e promover a vida. Além disso, ressalta-se que apesar da doença, a qualidade desse cuidado poderá prevenir reinternações ${ }^{13}$.

A falta de um protocolo sistematizado, elaborado especificamente para o tratamento de pessoas amputadas, é apontado em todas as entrevistas. Assim, apesar dos pacientes apresentarem-se com particularidades específicas, o procedimento de atendimento parece estruturar-se a partir da vivência de cada profissional. Portanto, considera-se que seguir um protocolo operacional padronizado e específico pode contribuir para a qualificação do atendimento ao paciente submetido à amputação.

\section{CONSIDERAÇÕES FINAIS}

Os resultados desta pesquisa demonstram que os profissionais entrevistados, bem como, os envolvidos no processo de atendimento aos pacientes amputados, procuram desempenhar suas atividades com esmero, dando atenção não somente aos aspectos físicos da recuperação, mas, também, aos aspectos psicológicos e emocionais. Porém, observa-se que a inexistência de um protocolo sistematizado de atendimento resulta em que cada profissional adote a estratégia que conhece para a superação das dificuldades decorrentes de suas atividades.

Assim, a definição de um protocolo sistematizado de atendimento integrado ao sistema de atendimento no pós-alta, pode qualificar esse atendimento, além de contribuir com a redução de reinternações e com ações de reabilitação direcionadas aos pacientes amputados.

A atenção aos aspectos psicológicos e emocionais revela preocupação da equipe, quanto à importância do papel do profissional que está em contato direto com os pacientes e familiares. Essa compreensão coletiva, demonstrada nas entrevistas, é essencial para um atendimento de qualidade. Entretanto, a infraestrutura física deve ser melhorada, qualificando o atendimento aos usuários.

Conclui-se, por fim, que apesar das dificuldades inerentes ao sistema de saúde pública no Brasil, estas são, em vários aspectos, mitigadas pelo esforço dos profissionais, em especial da equipe de enfermagem, envolvidos no atendimento direto ao paciente.

\section{Referências}

1 Smeltzer SC, Bare BG. Brunner \& Suddarth: tratado de enfermagem médico-cirugica. 12 ed. Rio de Janeiro: Guanabara Koogan; 2011.

2 Carvalho JA. Amputações de membros inferiores: em busca da plena reabilitação. 2. ed. São Paulo: Manole; 2003.

3 Schoeller SD, Silva DMG V, Vargas MAO, Borges AMF, Pires DEP, Bonetti A. Characteristics of amputated people cared for at a rehabilitation center. Journal of Nursing UFPE on line [internet]. 2013 fev [cited 2013 apr 10];7(2):445-51. Available from: http:// www.revista.ufpe.br/revistaenfermagem/index.php/revista/ article/viewFile/3351/pdf_2013 DOI: 10.5205/01012007

4 Minayo MCS. O desafio do conhecimento: pesquisa qualitativa em saúde. 14. ed. São Paulo: HUCITEC; 2014.

5 Resolução CNS N 196, de 10 de outubro de 1996 (BR). Institui Diretrizes e Normas regulamentadoras de pesquisas envolvendo seres humanos. [citado 2010 jun 30]. Available from: http://dtr2004.saude.gov.br/susdeaz/legislacao/arquivo/ Resolucao_196_de_10_10_1996.pdf

6 Gabarra LM, Crepaldi MA. Aspectos psicológicos da cirurgia de amputação. Aletheia [Internet]. 2009 [citado 2011 Nov 05];(30):59-72. Disponível em: http://pepsic. bvsalud.org/scielo.php?script=sci_arttext\&pid=S141303942009000200006\&lng=pt.

7 Chini GCO, Boemer MR. Amputation in the perception of those who experience it: a study under the phenomenological. Rev. Latino-Am. Enfermagem [Internet]. 2007 [citado 2011 Nov 11];15(2):330-336. Disponível em: http:// www.scielo.br/scielo.php?script=sci_arttext\&pid=S010411692007000200021\&lng=pt doi: 10.1590/S0104-

11692007000200021
8 Lucas LPP, Barichello E, Zuffi FB, Barbosa MH. A percepção dos portadores de Diabetes Mellitus tipo 2 em relação à amputação. Rev. Eletr. Enf. [Internet]. [citado 201306 03];2010;12(3):535-8. Available from: http://www.fen.ufg.br/revista/v12/n3/v12n3a17. htm. doi: 10.5216/ree.v12i3.6005

9 Gouveia GC, Souza WV, Luna CF, Souza-Júnior PRB, Szwarcwald CL. Satisfação dos usuários do sistema de saúde brasileiro: fatores associados e diferenças regionais. Rev. bras. epidemiol. [Internet]. 2009 Set [citado 2011 Nov 020];12(3):281-296. Disponível em: http://www.scielo.br/scielo.php?script=sci_ arttext\&pid=S1415-790X2009000300001\&lng=pt doi: $10.1590 /$ S1415-790X2009000300001

10 Matheus MCC, Pinho FS. Buscando mobilizar-se para a vida apesar da dor ou da amputação. Acta. Paul. Enferm. 2006;19(1):4955.

11 Chini GCO, Boemer MR. A amputação na percepção de quem a vivencia: um estudo sob a ótica fenomenológica. Rev Latino-am Enfermagem 2007 [cited 2013 jun 10]; 15(2): 330-336. Available from: http://www.scielo.br/pdf/rlae/v15n2/pt_v15n2a21

12 Martin LM. A ética e a humanização hospitalar. In: Pessini, L.; Bertachini, L. (Org.) Humanização e cuidados paliativos. São Paulo: Centro Universitário São Camilo/Loyola; 2004. p. 31-50. http://www. cesumar.br/epcc2009/anais/maria_ivoneide_silva.pdf

13 Foss MHDA, Martins MRI, Mazaro LM, Martins MID, Godoy JMP. Qualidade de vida dos cuidadores de amputados de membros inferiores. Rev Neurocienc. 2008;17(1):8-13.

14 Nogueira LCL. Gerenciando pela qualidade total na saúde. 4 ed. Belo Horizonte: Editora de Desenvolvimento Gerencial; 2014. 